\title{
Perspective
}

\section{Changing Trends in the Performance of Percutaneous Renal Biopsy from Nephrologist to Interventional Radiologist: A Single-Center Experience}

\author{
Stephen M. Korbet William L. Whittier Roger A. Rodby \\ Department of Medicine, Division of Nephrology, Rush University Medical Center, Chicago, IL, USA
}

\section{Keywords}

Percutaneous renal biopsy · Native kidney · Interventional radiology $\cdot$ Fellowship training

\begin{abstract}
Background: Percutaneous renal biopsy of native kidneys (PRB) has been an integral part of the practice of nephrology. However, over the past 30 years, PRB has transitioned from a procedure performed only by nephrologists to interventional radiologists (IRs). We surveyed practicing nephrologists completing training in our program to determine the clinical practice patterns of PRB. Methods: The 78 fellows completing the nephrology program at Rush University Medical Center from June 1984 through June 2017 were successfully contacted and surveyed regarding their opinion on adequacy of their training and whether they performed PRB in practice and if not or no longer, why. To evaluate for differences in the performance of PRB over time, a comparison of 4 periods of fellowship completion (i.e., 1984-1990, 19912000, 2001-2010, 2011-2017) was performed. Results: All 78 nephrologists felt they had been adequately trained to perform PRB. PRB was performed by 45 (58\%) nephrologists post-fellowship, but a significant decline was observed over
\end{abstract}

(c) 2018 S. Karger AG, Basel

E-Mail karger@karger.com www.karger.com/ajn the 4 periods of time from 1984 to 2017 ( 100 vs. 86 vs. 52 vs. $20 \%, p<0.0001)$. The primary reason that 33 nephrologists did not perform PRB was that it was too time consuming and IR was available to perform PRB. Of the 71 nephrologists still in practice only $12(17 \%)$ continue to perform PRB. A greater proportion of nephrologists completing training from 1984 1990 continue to perform PRB relative to those trained after 1990. The universal reason that nephrologists were no longer performing PRB was again an issue of time and the fact that IRs were available to perform PRB. Conclusion: We find that there has been a significant transition over time in the performance of PRB by a nephrologist to IR. The major reason for this is the time burden associated with PRB and the availability of IRs.

(c) 2018 S. Karger AG, Basel

\section{Introduction}

The percutaneous renal biopsy of native kidneys (PRB) has been an integral part of research and the clinical practice of nephrology and as such has been a cornerstone in the training of future nephrologists during their fellowship. However, over the last 30 years, we have witnessed 
Fig. 1. Performance of $\mathrm{PRB}$ post-fellowship based on the year of fellowship completion $(p<0.0001)$.

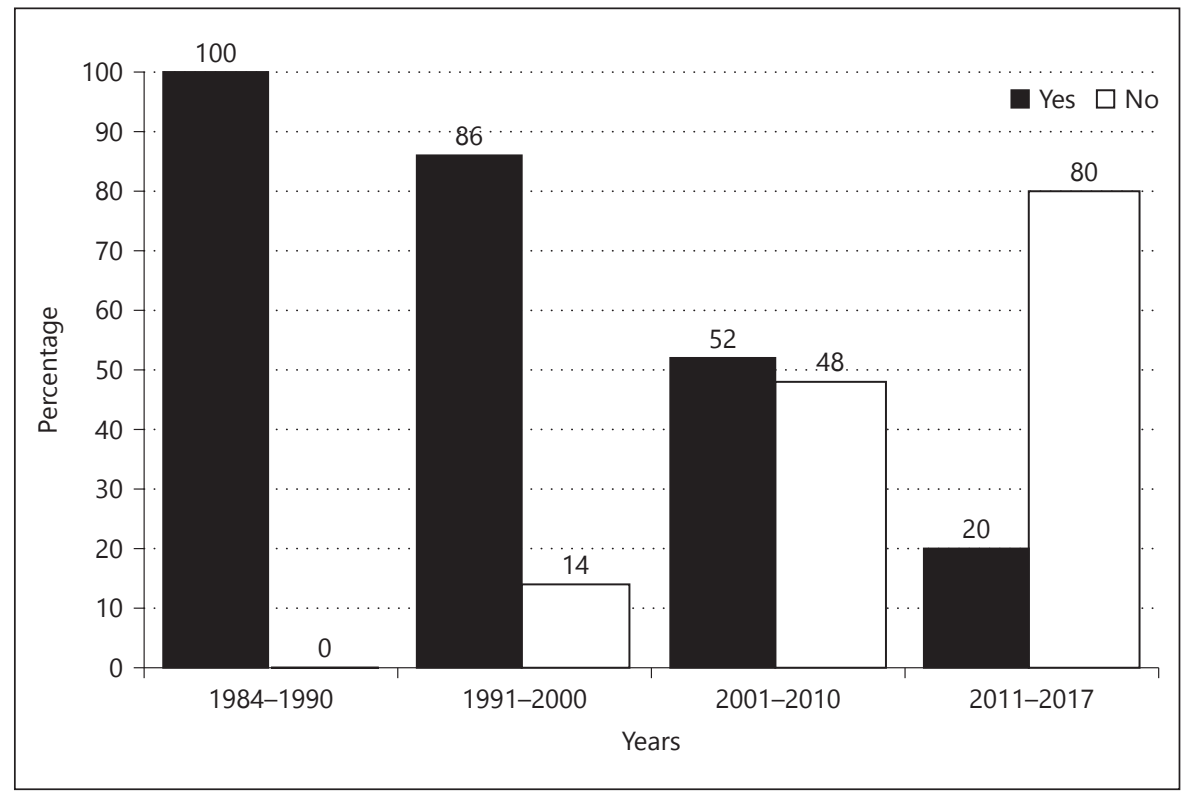

the steady transitioning of a procedure once performed only by nephrologists to one that is performed predominantly by interventional radiologists (IRs) [1-7]. The reason for this changing trend has been attributed to a number of factors including inadequate training as well as issues related to reimbursement, liability and time commitment in the face of ever increasing workloads [1, $2,6]$. As fewer and fewer nephrologists perform PRB, the issue of whether competence in performing PRB should remain a requirement in certification by the American Board of Internal Medicine has come into question [1, 8, 9]. We surveyed practicing nephrologists who completed training in our program from 1984 through 2017 to better determine the clinical practice patterns of PRB.

\section{Methods}

The nephrology fellowship program at Rush University Medical Center has trained fellows to perform PRB of native kidneys since June 1983 using real-time ultrasound imaging performed by an experienced radiologist [10]. The fellows perform all PRBs under the supervision of an attending nephrologist who is also competent to perform PRB. A total of 78 fellows completed our program from June 1984 through June 2017 and went on to practice nephrology. All became board certified. In order to determine the post-training PRB practice patterns of our nephrologists, we contacted all 78 and all agreed to answer the following questions:

1. Did you feel adequately trained to perform PRB by the end of your fellowship?

2. Did you perform PRB post-fellowship?

If yes, for how many years?

Changing Trends in the Performance of

PRB
If yes, are you still performing PRB and if not, why (a time, reimbursement or liability issue)?

If no, why (a time, reimbursement or liability issue)?

3. Are you still a practicing nephrologist?

The overall proportion of fellows performing PRB after entering practice, the duration they performed $\mathrm{PRB}$ and the proportion of currently practicing nephrologists still performing PRB were assessed and compared. To evaluate for possible differences in the performance of PRB over time, a comparison of 4 periods of fellowship completion (i.e., 1984-1990, 1991-2000, 2001-2010, 2011-2017) was performed. This study was approved by the institutional review board.

Statistical analysis was performed using the Mann-Whitney test or Kruskal-Wallis test for continuous data or the Fisher's exact test or chi-square test for categorical data using GraphPad InStat version 3.06 for Windows (GraphPad Software, San Diego, CA, USA, www.graphpad.com). Data are reported as mean \pm SD and a $p$ value of $<0.05$ was considered significant.

\section{Results}

All 78 nephrologists felt they had been adequately trained to perform PRB by the completion of their fellowship. PRB of native kidneys was performed by 45 (58\%) of the 78 nephrologist immediately post-fellowship, but a significant decline was observed in the performance of PRB by nephrologists completing training over the 4 periods from 1984 to 2017 ( $8 / 8$ vs. $18 / 21$ vs. $15 / 29$ vs. $4 / 20$, $p<0.0001$; Fig. 1). Overall, nephrologists who performed PRB did so for $11 \pm 9$ years and they performed PRB for $\geq 1$ year in $100 \%$ of cases, $\geq 5$ years in $32 / 45$ (71\%) of cases 
and $\geq 10$ years in $23 / 45$ (51\%) of cases. Nephrologists performing PRB were in practice longer than those nephrologists not performing PRB (18 \pm 8 vs. $8 \pm 5$ years, $p<$ $0.0001)$.

All 33 fellows not doing PRB referred patients to IR for $\mathrm{PRB}$ at their institutions. The primary reason for not performing biopsies provided by all 33 nephrologists was that PRB was too time consuming and IR was available and willing to do the procedure at their institutions. The issue of liability was expressed as a factor by 17 out of 33 (52\%) but only 10 out of $33(30 \%)$ of nephrologists expressed that reimbursement was a factor in not performing PRB.

A significant decrease in the performance of PRB by nephrologists was observed during the time from entering practice to the present. Of the 71 nephrologists still in practice (7 retired, 5 having previously performed $\mathrm{PRB}$ ), only 12 ( $17 \%$ vs. $45 / 78-58 \%, p<0.0001)$ were still performing PRB. A greater proportion of nephrologists completing training from 1984 to 1990 continue to perform PRB relative to those completing training after 1990 (3/7$43 \%$ vs. $9 / 64-14 \%, p=0.08$ ). The reason for no longer performing PRB was again universally an issue of "time" and the fact that IR was available to perform the procedure.

\section{Discussion}

Our study confirms the steady transition in the performance of PRB from nephrologist to IR over the past 30 years. In 1990, Tape et al. [4] published a survey of 516 practicing nephrologists trained from 1969 to 1979 and found that $95 \%$ of board certified nephrologists performed PRB. However, 5 years after this report, a memorandum from the Renal Physician's Association found that $35 \%$ of percutaneous renal biopsies were being performed by radiologists. A study by Lane et al. [3], in 2011 noted that only $55 \%$ of PRBs were being performed by nephrologists. And, in 2012, a further decline was observed by Tondel et al. [5] who reported that from 1988 to 2010 only $35 \%$ of PRBs were performed by a nephrologist with a steady transitioning of PRB from nephrologist to IR during the 22-year period. Most recently, Yuan et al. [6], in a survey of 55 practicing nephrologists trained at Walter Reed National Military Medical Center from 1985 to 2017 found that while $83 \%$ felt they were adequately prepared, only $35 \%$ performed PRB. They found that $65 \%$ nephrologists in practice $\leq 10$ years had never performed a PRB or stopped within 5 years. Similarly Berns et al. [1] found that only $20 \%$ of 30 nephrologists trained in the
University of Pennsylvania Health System fellowship program in the last 6 years were performing PRB.

The reasons for the decreased performance of PRB by nephrologists have been attributed to inadequate training $[1,2,6]$ as well as issues related to reimbursement, liability, and time commitment in the face of an ever increasing workload $[2,6]$. However, it seems difficult to attribute the decrease on "inadequate training" when over $80 \%$ of fellows in the study by Yuan et al. [6] and 100\% of our past fellows expressed the feeling that they had been adequately prepared to perform PRB. The overriding reason for a nephrologist from our program not performing PRB has been the issue of time and convenience. Even in the Walter Reed nephrology program, the complex and timeconsuming process of coordinating and performing the PRB has resulted in their referring PRBs to IR [6]. Since 2012, their fellows have been trained to do PRB by IRs, as their nephrology faculty no longer performs the procedure [6].

The changing trend in the performance of PRB from nephrologists to IR is a reality. As nephrologists we have a vested interest in the biopsies we perform that is not shared by radiologists as the information provided by this procedure directly affects the care we provide our patients. Studies comparing PRB being done by nephrologists and IR have shown similar success and complication rates $[5,7]$. However, IR often perform PRBs using smaller gauge needles, which can result in a smaller sample size and this stands to jeopardize the diagnostic accuracy, which can have major implications in the management of our patients $[5,7]$.

The PRB has been an important part of the evolution of our subspecialty, research and the clinical practice of nephrology. We should try to devise a way to recapture PRB in order to ensure the ongoing optimal care of our patients. The American Society of Diagnostic and Interventional Nephrology, the interventional branch of nephrology, includes in their bylaws the performance of PRB as part of their scope, but their primary focus has been on dialysis access procedures. Thus, an option for interventional nephrologists would be to aggressively pursue the performance of PRB. An additional option is for nephrology programs performing $\mathrm{PRB}$ at major medical centers to become "renal pathology centers of excellence", developing an easy to use program, which would attract referrals for PRB from community nephrologists who would prefer to have the biopsies done by a nephrologist. This would ensure not only that an adequate sample would be obtained but that the tissue would be processed appropriately and read by an experienced nephropathologist. 
The fact that nephrologists are no longer performing biopsies once in clinical practice has led to a debate as to whether or not our fellows should even be required to be "competent" in performing PRB in order to qualify for taking the certifying exam $[1,6,8,9]$. Clearly nephrology fellows need to be competent regarding the indications, contraindications, and complications associated with a PRB. Whether they should be competent in performing a procedure they may never do again after training is a complex issue. In our program, we believe it is important that our fellows are trained to perform PRB, but clearly this is not universally agreed upon $[1,6,8,9]$. However, we feel that whether a nephrologist or IR does the PRB, it is the nephrologist's obligation to their patient to ensure that the procedure is done safely, that an adequate sample is obtained and processed appropriately, and that it is read by an experienced nephropathologist.

\section{Disclosure Statement}

The authors declared that they have no conflicts of interest to disclose. The results presented in this paper have not been published previously in whole or part, except in abstract format.

\section{References}

1 Berns JS: Training nephrology fellows in temporary hemodialysis catheter placement and kidney biopsies is needed and should be required. Clin J Am Soc Nephrol 2018;13:1099-1101.

2 Korbet SM: Nephrology and the percutaneous renal biopsy: a procedure in jeopardy of being lost along the way. Clin J Am Soc Nephrol 2012;7:1545-1547.

3 Lane C, Brown M: Alignment of nephrology training with workforce, patient, and educational needs: an evidence based proposal. Clin J Am Soc Nephrol 2011;6:2681-2687.
4 Tape TG, Wigton RS, Blank LL, Nicolas JA: Procedural skills of practicing nephrologists. A national survey of 700 members of the American College of Physicians. Ann Int Med 1990;113:392-397.

5 Tondel C, Vikse BE, Bostad L, Svarstad E: Safety and complications of percutaneous kidney biopsies in 715 children and 8573 adults in Norway 1988-2010. Clin J Am Soc Nephrol 2012;7:1591-1597.

6 Yuan CM, Nee R, Little DJ, et al: Survey of kidney biopsy clinical practice and training in the United States. Clin J Am Soc Nephrol 2018;13:718-725.

7 Whittier WL, Korbet SM: Who should perform the percutaneous renal biopsy: a nephrologist or radiologist? Semin Dial 2014;27: 243-245.
8 Brown RS: Kidney biopsy training and the future of nephrology: what about the patient? Clin J Am Soc Nephrol 2018;13:11051106.

9 Norby SM: Requirements for procedural skills in nephrology training programs: framing the conversation. Clin J Am Soc Nephrol 2018;13:1096-1098.

10 Korbet SM, Volpini KC, Whittier WL: Percutaneous renal biopsy of native kidneys: a single-center experience of 1,055 biopsies. Am J Nephrol 2014;39:153-162. 